\title{
Predictive value of SAPS II and APACHE II scoring systems for patient outcome in a medical intensive care unit
}

\author{
Amina Godinjak ${ }^{1}$, Amer Iglica ${ }^{2}$, Admir Rama $^{3}$, Ira Tančica ${ }^{4}$, Selma Jusufović 5 , \\ Anes Ajanović ${ }^{4}$, Adis Kukuljac ${ }^{1}$
}

\author{
${ }^{1}$ Medical intensive care unit, Clinical \\ Center University of Sarajevo, Bosnia \\ and Herzegovina, ${ }^{2}$ Clinic for heart \\ vascular diseases and rheumatology \\ Clinical Center University of Sarajevo \\ Bosnia and Herzegovina, ${ }^{3}$ Bahceci IVF \\ Center Sarajevo, Bosnia and Herzegovina \\ ${ }^{4}$ Clinic for anesthesiology, Clinical \\ Center University of Sarajevo, Bosnia and \\ Herzegovina, ${ }^{5} \mathrm{Clinic}$ for endocrinology \\ Clinical Center University of Sarajevo \\ Bosnia and Herzegovina \\ Correspondence: \\ aminagodinjak@gmail.com \\ Tel.: + 38733297079 \\ Fax.: + 38733297813
}

Received: 6 April 2016

Accepted: 30 September 2016

Key words: SAPS II • APACHE II • Medical intensive care unit.

\begin{abstract}
Objective. The aim is to determine SAPS II and APACHE II scores in medical intensive care unit (MICU) patients, to compare them for prediction of patient outcome, and to compare with actual hospital mortality rates for different subgroups of patients. Methods. One hundred and seventy-four patients were included in this analysis over a oneyear period in the MICU, Clinical Center, University of Sarajevo. The following patient data were obtained: demographics, admission diagnosis, SAPS II, APACHE II scores and final outcome. Results. Out of 174 patients, 70 patients $(40.2 \%)$ died. Mean SAPS II and APACHE II scores in all patients were $48.4 \pm 17.0$ and $21.6 \pm 10.3$ respectively, and they were significantly different between survivors and non-survivors. SAPS II $>50.5$ and APACHE II $>27.5$ can predict the risk of mortality in these patients. There was no statistically significant difference in the clinical values of SAPS II vs APACHE II ( $p=0.501)$. A statistically significant positive correlation was established between the values of SAPS II and APACHE II ( $r=0.708 ; \mathrm{p}=0.001)$. Patients with an admission diagnosis of sepsis/septic shock had the highest values of both SAPS II and APACHE II scores, and also the highest hospital mortality rate of $55.1 \%$. Conclusion. Both APACHE II and SAPS II had an excellent ability to discriminate between survivors and non-survivors. There was no significant difference in the clinical values of SAPS II and APACHE II. A positive correlation was established between them. Sepsis/septic shock patients had the highest predicted and observed hospital mortality rate.
\end{abstract}

\section{Introduction}

There are many intensive care unit (ICU) scoring systems, and many new ones are being developed to achieve an objective and quantitative description of the degree of organ dysfunction and evaluation of morbidity in ICU patients. Scoring systems such as: Acute Physiology and Chronic Health Evaluation (APACHE) II, III and IV, Sim- plified Acute Physiology Score (SAPS), Sepsis-related Organ Failure Assessment Score (SOFA), Mortality Prediction Model (MPM), Multiple Organ Dysfunction Score (MODS), and Logistic Organ Dysfunction Score (LODS) have become a necessary tool to describe ICU populations and to explain differences in mortality (1).

The Acute Physiology and Chronic Health Evaluation II (APACHE II) is the 
most commonly used severity-of-disease scoring system in ICUs around the world (2). Within the first 24 hours of patient admittance, the worst value for each physiological variable is calculated into an integer score from 0 to 71 . Higher scores represent a more severe disease and a higher hospital mortality risk. The first APACHE model was presented by Knaus et al. in the 1980's (3). It has not been validated for use in patients under the age of 16. Even though newer scoring systems have been developed, APACHE II still continues to be used because so much documentation is based on it. The relationship between APACHE II scores and approximate mortality interpretation in medical (non-surgical) patients is shown in Table 1.

The Simplified Acute Physiology Score II (SAPS II) was first described in 1984 as an alternative to the APACHE scoring system (4). The SAPS II score is calculated from the

Table 1 APACHE II score and hospital mortality interpretation for non-surgical patients (3)

\begin{tabular}{ll}
\hline APACHE II score & Hospital mortality* \\
\hline $0-4$ & $4 \%$ \\
$5-9$ & $8 \%$ \\
$10-14$ & $15 \%$ \\
$15-19$ & $24 \%$ \\
$20-24$ & $40 \%$ \\
$25-29$ & $55 \%$ \\
$30-34$ & $73 \%$ \\
$35-100$ & $85 \%$ \\
\hline & *Approximate interpretation (non-surgical patients).
\end{tabular}

Table 2 SAPS II score and hospital mortality interpretation (4)

\begin{tabular}{ll}
\hline SAPS II score (points) & Mortality \\
\hline 29 & $10 \%$ \\
40 & $25 \%$ \\
52 & $50 \%$ \\
64 & $75 \%$ \\
77 & $90 \%$ \\
\hline
\end{tabular}

worst value of 12 routine physiological measurements during the first 24 hours of patient admisson, information about previous health status and some information obtained at admission. 24 hours after admission to the ICU, the measurement is completed and this results in an integer point score between 0 and 163 , and predicted hospital mortality between $0 \%$ and $100 \%$. There is a sigmoidal relationship between SAPS II score and mortality rate. SAPS II score and mortality rate interpretation are shown in Table 2.

Previous studies have reported the varying performance of these scoring systems in predicting hospital mortality. Several studies had reported better performance by APACHE II $(5,6)$. Other studies on different patient populations validated SAPS II as a good prediction scoring system $(7,8)$. Juneja et al. (9) reported that the difference in performance of the scoring systems was not significant and depends on local preferences. Newer scoring systems have been developed, such as APACHE III and IV, to refine the previous APACHE II. However, APACHE II and SAPS II as the simplest and inexpensive scoring systems are still used in our medical intensive care unit (MICU).

The aim of this study is to determine the SAPS II and APACHE II scores in patients admitted to the MICU and compare them for prediction of the outcome in these patients (survivors i.e. patients who were discharged from the hospital, and non-survivors i.e. patients who died during the same hospitalization). Predictive scores were calculated and actual hospital mortality rates were compared for different subgroups of MICU patients.

\section{Materials and methods}

\section{Study design and data collection}

The Clinical Center of the University of Sarajevo is a 1952-bed tertiary university hospital, with a 7-bed closed MICU with a 
nurse/patient ratio of 1:3.5. One hundred and eighty-nine (189) patients were admitted to the MICU in the Clinical Center of the University of Sarajevo from October 2014 to September 2015. Patients were either admitted from the emergency department or transferred from a hospital ward. There were 15 patients who had exitus letalis in the first 24 hours of hospitalization in the MICU, for which SAPS II and APACHE II scores could not be calculated. The remaining 174 patients that were hospitalised for more than 24 hours in the MICU were included in the study. Out of 174 patients, 89 were admitted from the emergency department and 85 were transferred from a hospital ward. For patients with multiple admissions, only the first data set was included in the data analysis. For each patient the following data were obtained: demographic data, admission diagnosis, parameters for SAPS II and APACHE II scores and the final outcome (survivors i.e. patients who were discharged from the hospital, and non-survivors i.e. patients who died during the same hospitalization). The reasons for admission were grouped into five categories: sepsis / septic shock (based on the presence of systemic inflamatory response syndrome and a source of infection with/without hypotension and hypoperfusion despite adequate fluid resuscitation), respiratory failure (hypoxemia and/or hypercarbia requiring non-invasive ventilation or me.chanical ventilation), cardiovascular (based on clinical, laboratory and ECG and/ or echocardiography findings), neurological (based on clinical and diagnostic findings of central nervous system damage) and other causes. Surgical, burns, coronary care and cardiac surgery patients were not admitted to this MICU. When multiple diagnoses were present, the leading one, with the worst prognosis was selected as the main reason for admission. SAPS II and APACHE II were calculated 24 hours after admission to the MICU. The SAPS II score was cal- culated from the following parameters: age, heart rate, systolic blood pressure, temperature, Glasgow Coma Scale, mechanical ventilation or continuous positive airway pressure (CPAP), $\mathrm{PaO} 2 / \mathrm{FiO}_{2}$, urine output, urea, sodium, potassium, bicarbonate, bilirubin, leucocyte count, chronic diseases, type of admission. The APACHE II score was calculated from the patient's age and 12 parameters: $\mathrm{PaO}_{2}$, temperature, mean arterial pressure, arterial $\mathrm{pH}$, heart rate, respiratory rate, sodium, potassium, creatinine, hematocrit, leucocyte count and Glasgow Coma Scale. Also, information about previous health status (surgery, history of organ insufficiency, immunocompromised state) was calculated into the result. The worst parameters in the first 24 hours of hospitalization were selected for calculation of the scores.

\section{Statistical analysis}

Data were presented for continuous variables as means and standard deviation, and for categorical variables as absolute and relative frequencies. The data were analysed using t-test, Fisher's exact and chi-square test. A receiver operating characteristic (ROC) curve was used to determine a cut-off value for mortality, and the sensitivity and specificity of each scoring system for prediction of mortality. Pearson's correlation was used for evaluating the correlation between the scoring systems. Statistical significance was interpreted as $\mathrm{p} \leq 0.05$. Graphically, data were presented in the form of tables and figures. Data were analysed using SPSS for Windows version 20.0 (SPSS Inc., Chicago, IL, USA).

\section{Results}

Out of 174 patients included in the study, 104 patients (59.8\%) survived and 70 patients $(40.2 \%)$ died. One hundred patients were male $(57.5 \%)$. Their mean age was $61.7 \pm 16.3$ years (range 19-87). When survi- 
vors v. non-survivors were compared, there was no statistical difference in patient age (59.6 \pm 14.7 vs. $62.3 \pm 15.9 ; \mathrm{p}=0.654)$ or gender $(61$ males vs. 43 females in the survivor group and 39 males vs. 31 females in the non-survivor group; $\mathrm{p}=0.232$ ). Mean SAPS II and APACHE II scores in all admitted patients were $48.4 \pm 17.0$ and $21.6 \pm 10.3$ respectively. The mean SAPS II score was $41.2 \pm 14.1$ in survivors and 63.9 \pm 11.2 in non-survivors $(\mathrm{p}<0.0001)$. The mean APACHE II score was $16.9 \pm 6.4$ in survivors and $31.5 \pm 10.2$ in nonsurvivors $(p<0.0001)$. The receiver operating characteristics curve results of SAPS II and APACHE II scores in prediction of fatal outcome are shown in Figure 1.

The area under the ROC curve was calculated to evaluate the predictive value of the scoring systems. The SAPS II scoring system represents a statistically significant predictive marker of fatal outcomes of patients (area under the curve of 0.892, CI 0.84-0.94, $\mathrm{p}=0.001$ ). Cut off value for SAPS II was 50.5, with the sensitivity of $90.2 \%$ and specificity of $75.7 \%$. The APACHE II scoring system represents a statistically significant predictive marker of fatal outcomes of patients (area under the curve of 0.920, CI 0.87-0.97, $\mathrm{p}=0.001$ ). Cut off value for APACHE II was 27.5 , with sensitivity of $74.5 \%$ and specificity of $93.4 \%$. When the ROC curves were compared, that there was no statistically significant difference in the clinical values of SAPS II vs APACHE II ( $\mathrm{p}=0.501$ ). By using Pearson's correlation, a statistically significant positive correlation was established between the values of SAPS II and APACHE II $(r=0.708 ; p=0.001)$. This means that when SAPS II value increases, so does the value of APACHE II, as shown in Figure 2.

SAPS II and APACHE II scores and hospital mortality for patients based on their admission diagnoses, are shown in Table 3.

Respiratory failure was the leading cause for ICU admission (62 patients, 35.6\%), followed by sepsis /septic shock (49 patients, $28.1 \%$ ) and cardiovascuar failure (31 patients, 17.8\%). Patients with admission diagnosis of sepsis/septic shock had the highest values of both SAPS II and APACHE II scores, and also the highest hospital mortality rate of $55.1 \%$. Patients in the group admitted for other causes had the lowest SAPS II score. Patients with respiratory failure had the lowest APACHE II score and lowest hospital mortality rate.
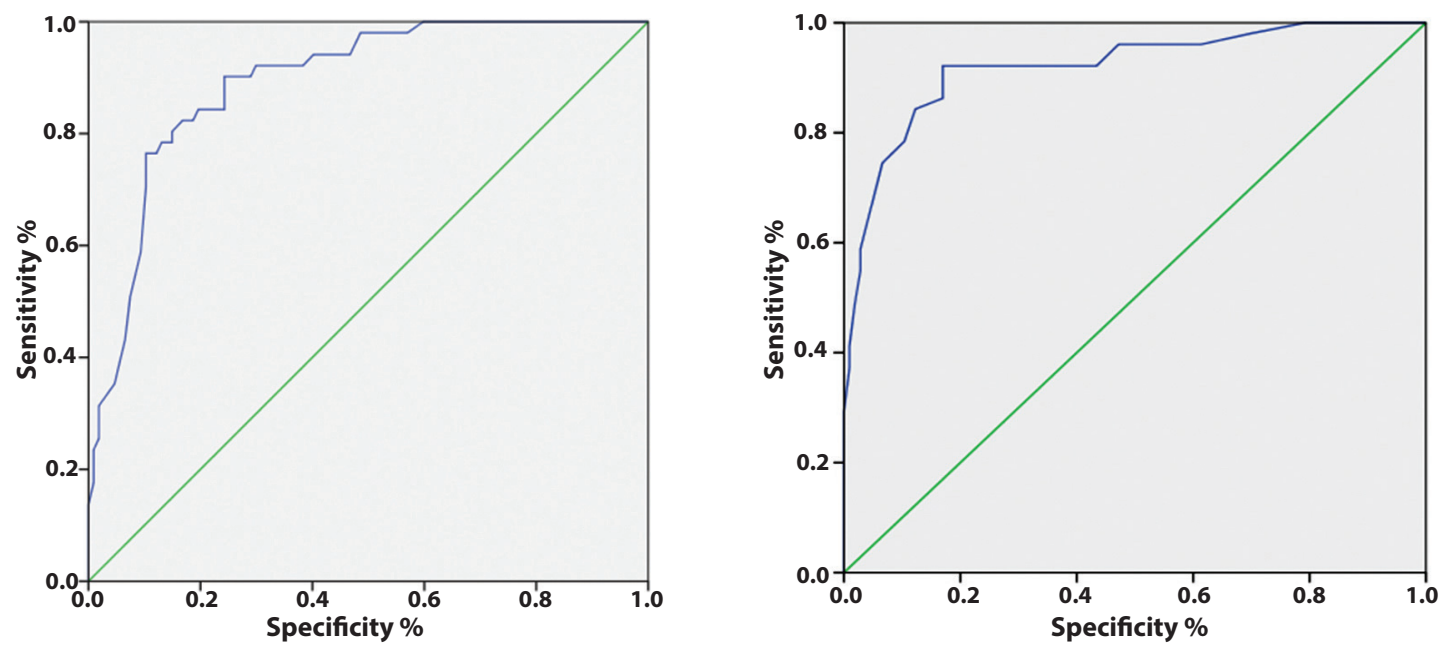

Figure 1 Receiver operating curve for predicting fatal outcome according to SAPS II and APACHE II scoring systems. 


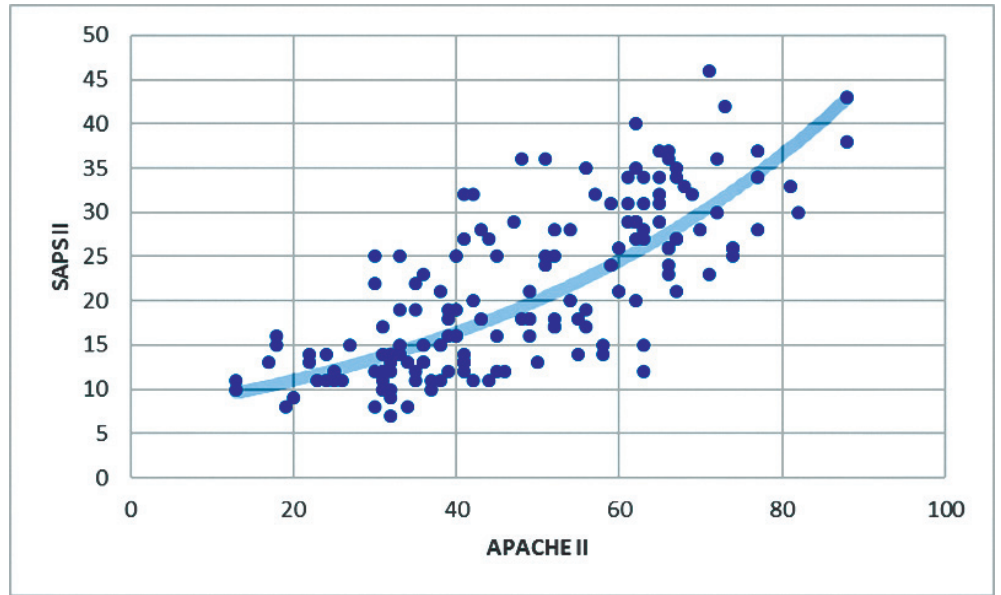

Figure 2 Correlation between SAPS II and APACHE II scores.

Table 3 Predictive scoring systems and hospital mortality rate in studied patients according to admission diagnosis

\begin{tabular}{|c|c|c|c|c|c|}
\hline \multirow[b]{2}{*}{ Characteristics } & \multicolumn{5}{|c|}{ Patients admitted to the ICU with the primary admission diagnosis $(n=174)$} \\
\hline & $\begin{array}{l}\text { Respiratory } \\
\text { failure } \\
(n=62)\end{array}$ & $\begin{array}{l}\text { Sepsis/septic } \\
\text { shock } \\
(n=49)\end{array}$ & $\begin{array}{l}\text { Cardiovascular } \\
\text { failure } \\
(n=31)\end{array}$ & $\begin{array}{l}\text { Neurological } \\
\text { causes } \\
(n=16)\end{array}$ & $\begin{array}{l}\text { Other } \\
\text { causes } \\
(n=16)\end{array}$ \\
\hline SAPS II $(\bar{x} \pm S D)$ & $42.4 \pm 14.9$ & $58.4 \pm 15.0$ & $47.6 \pm 17.8$ & $55.4 \pm 17.0$ & $40.9 \pm 15.0$ \\
\hline APACHE II $(\bar{x} \pm S D)$ & $17.5 \pm 7.5$ & $27.0 \pm 11.9$ & $21.5 \pm 9.8$ & $26.0 \pm 11.0$ & $20.1 \pm 9.1$ \\
\hline Hospital mortality rate (\%) & 17.7 & 55.1 & 51.6 & 50.0 & 25.0 \\
\hline
\end{tabular}

\section{Discussion}

According to our results, a SAPS II score higher than 50.5 can predict the MICU patients' mortality rate with good sensitivity (90.2\%) and lower specificity (75.7\%). An APACHE II score higher than 27.5 can predict the MICU patients' mortality rate with good specificity (93.4\%) but with lower sensitivity (74.5). Based on further analysis, there was no significant difference in the clinical values of SAPS II and APACHE II. Haq et al. (10) also showed similar performance by SAPS II and APACHE II. Sathe et al. (11) and Mosenson et al. (12) showed that APACHE II had better discrimination than SAPS II.

APACHE II was a less sensitive predictor than SAPS II, but with higher specificity. The specificity of APACHE II was $93.4 \%$ which is higher than reported by Sekulic et al. (13).
In a study of 11,300 patients from 35 hospitals in California, the authors noted that only the APACHE II scoring system showed good discrimination for predicting ICU mortality (14). However, Sekulic et al. (13) concluded that SAPS II was a better predictor for hospital mortality in ICU patients. In a study by Ho et al. (15) SAPS II was confirmed to have the best performance overall.

As different subpopulations of critically ill patients are admitted to our MICU every day, there was a need to evaluate which subgroup had the worst expected and actual prognosis. The way in which the patients were divided is in accordance with the study by Breslow et al. where the diagnosis was documented within the first ICU day; it reflected the primary reason for ICU admission; and, when multiple diagnoses were relevant, the diagnosis with the worst prognosis (e.g., sepsis rather than hyper- 
glycemia) was the leading one (16). When our patients were divided into subgroups according to their admission diagnosis, the leading cause of admission was respiratory failure, followed by sepsis / septic shock. Severe sepsis and septic shock were shown to be major reasons for ICU admission and also the leading causes of mortality in noncoronary ICUs (17). Hospital mortality in patients with sepsis was $55.1 \%$ which is in accordance with the results by Mohan et al. study (18). In our study, patients with respiratory failure had a hospital mortality rate of $17.7 \%$ which is lower than $30.7 \%$ as reported by Evran et al. (19).

Juneja et al. (9) indicated that the newer scoring systems performed better than their older counterparts, and were more accurate. Nevertheless, the difference in performance was not statistically significant and the choice of scoring system may depend on the ease of use and local preferences.

\section{Limitations of study}

The present study has some limitations. First, our small sample size is a limiting factor in analysis. Also, being a single center study, there is possibly some amount of bias due to differences in ICU admission policies.

\section{Advantages of study}

The results of this study and of past studies suggest ambiguous and inconclusive results regarding outcome, and they are heavily dependent on patient populations and medical interventions used on those patients. This must be taken into consideration when it comes to the interpretation of results. The existence of a large number of scoring systems suggests that the ideal model has yet to be found. Differences in the performance of scoring systems reinforce the need to validate them using data from independent samples from different ICUs in different countries due to variations in the structure and quality of medical care, as well as genetic differences between populations.

\section{Conclusion}

In conclusion, both APACHE II and SAPS II have an excellent ability to discriminate between survivors and non-survivors. ROC curve analysis showed that there was no significant difference in the clinical values of SAPS II vs APACHE II in MICU patients. Also, a positive correlation was established between the values of SAPS II and APACHE II scores. Sepsis/septic shock patients had the highest predicted and observed hospital mortality rate.

\section{What is already known on this topic}

Scoring systems predict patient outcomes in intensive care units. Over the years, many studies have evaluated the predictive ability of various scoring systems, and conflicting data have been reported so far. Although there are newer scoring systems, some of the most widely used are SAPS II and APACHE II. There is a need for more studies evaluating various scoring systems to predict mortality in different patient populations.

What this study adds

To the best of our knowledge, this is the first study to evaluate predictive scoring systems of patient outcome in a medical in tensive care unit in Bosnia and Herzegovina.

Authors' contributions: Conception and design: AG and SJ; Acquisition, analysis and interpretation of data: AK, IT and AR; Drafting the article: AA and AG; Revising it critically for intellectual content: AI and AK; Approved final version of the manuscript: AG and IT.

Conflict of interest: The authors declare that they have no conflict of interest.

\section{References}

1. Rapsang AG, Shyam DC. Scoring systems in the intensive care unit: A compendium. Indian J Crit Care Med. 2014;18(4):220-8.

2. Salluh JI, Soares M. ICU severity of illness scores: APACHE, SAPS and MPM. Curr Opin Crit Care. 2014;20(5):557-65. 
3. Knaus WA, Draper EA, Wagner DP. APACHE II: a severity of disease classification system. Crit Care Med. 1985;13(10):818-29.

4. Le Gall JR, Lemeshow S, Saulnier F. A new Simplified Acute Physiology Score (SAPS II) based on a European/North American multicenter study. JAMA. 1993;270(24):2957-63.

5. Arabi Y, Haddad S, Goraj R, Al-Shimemeri A, Al-Malik S. Assessment of performance of four hospital hospital mortality prediction systems in a Saudi Arabian intensive care unit. Crit Care. 2002;6(2):166-74.

6. Khwannimit B, Geater A. A comparison of APACHE II and SAPS II scoring systems in predicting hospital hospital hospital mortality in Thai adult intensive care units. J Med Assoc Thai. 2007;90(4):643-52.

7. Gupta R, Arora VK. Performance evaluation of APACHE II score for an Indian patient with respiratory problems. Indian J Med Res. 2004;119(6):273-82.

8. Tempe A, Wadhwa L, Gupta S, Bansal S, Satyanarayana L. Prediction of hospital hospital mortality and morbidity by simplified acute physiology score II in obstetric intensive care unit admissions. Indian J Med Sci. 2007;61(4):179-85.

9. Juneja D, Singh O, Nasa P, Dang R. Comparison of newer scoring systems with the conventional scoring systems in general intensive care population. Minerva Anestesiol. 2012;78(2):194-200.

10. Haq A, Patil S, Parcells AL, Chamberlain RS. The Simplified Acute Physiology Score III Is Superior to the Simplified Acute Physiology Score II and Acute Physiology and Chronic Health Evaluation II in Predicting Surgical and ICU Mortality in the "Oldest Old". Curr Gerontol Geriatr Res. 2014;2014:934852.

11. Sathe PM, Bapat SN. Assessment of performance and utility of mortality prediction models in a sin- gle Indian mixed tertiary intensive care unit. Int J Crit Illn Inj Sci. 2014;4(1):29-34.

12. Moseson EM, Zhuo H, Chu J, Stein JC, Matthay MA, Kangelaris KN, et al. Intensive care unit scoring systems outperform emergency department scoring systems for mortality prediction in critically ill patients: a prospective cohort study. J Intensive Care. 2014;2:40.

13. Sekulic AD, Trpkovic SV, Pavlovic AP, Marinkovic OM, Ilic AN. Scoring systems in assessing survival of critically ill ICU patients. Med Sci Monit. 2015;21:2621-9.

14. Vasilevskis EE, Kuzniewicz MW, Cason BA, Lane RK, Dean ML, Clay T, et al. Mortality probability model III and simplified acute physiology score II: assessing their value in predicting length of stay and comparison to APACHE IV. Chest. 2009;136(1):89-101.

15. Ho K, Lee K, Williams T, Finn J, Knuiman M, Webb SA. Comparison of acute physiology and chronic health evaluation (APACHE) II score with organ failure scores to predict hospital mortality. Anaesthesia. 2007;62(5):466-73.

16. Breslow JM, Badawi O. Severity scoring in the critically ill (part 1 - interpretation and accuracy of outcome prediction scoring systems) Chest. 2012;141(1):245-52.

17. Dellinger RP, Levy MM, Rhodes A. Surviving sepsis campaign: international guidelines for management of severe sepsis and septic shock: 2012. Crit Care Med. 2013;41(2):580-637.

18. Mohan A, Shrestha P, Guleria R, Pandey RM, Wig N. Development of a mortality prediction formula due to sepsis/severe sepsis in a medical intensive care unit. Lung India. 2015;32(4):313-9.

19. Evran T, Serin S, Gürses E, Sungurtekin H. Various scoring systems for predicting mortality in Intensive Care Unit. Niger J Clin Pract. 2016;19(4):530-4. 